\title{
The magnification invariant of simple galaxy lens models
}

\author{
N. Dalal \\ Physics Dept., University of California, San Diego, La Jolla, CA 92093 USA
}

\begin{abstract}
We demonstrate that for several of the gravitational lens models used to describe galaxies, there exists a quantity we dub the magnification invariant, equaling the sum of the signed magnifications of the images, that is a constant when the image multiplicity is a maximum. This invariant is independent of most of the model parameters, and is independent of the source position as long as the source lies inside the caustic. It is not necessary to solve the lens equations to compute this invariant. For quad lenses, it is usually easy to assign image parities and thus one can compute the sum of the signed fluxes, compare with the magnification invariant of different models, and infer the model magnification factors of the images without fitting. We evaluate the invariant for simple galaxy models, apply it to known cases of quadruple lenses, and discuss implications for the ability of these lens models to reproduce observed image brightnesses.
\end{abstract}

Subject headings: gravitational lensing-galaxy models-individual(CLASS 1608+656, H1413+117). 


\section{INTRODUCTION}

Gravitational lensing is a well-known phenomenon that has emerged as a useful tool in modern astrophysics. Two important classes of gravitational lenses are galaxy lenses, which can produce multiple images of the background source with typical image separations of arcseconds (Keeton and Kochanek, 1996), and lensing by compact objects in our own and nearby galaxies (eg Alcock et al., 1997 and Alcock et al., 1998). The latter class, termed "microlenses" since typical image separations are too small to permit individual image resolution, are modeled as point masses. Models of galaxy lenses can be much more complicated, since the mass distributions of galaxies, although not well understood, are certainly far more complex than those of the starlike microlenses. Since galaxies are thought to be dominated by dark halos in roughly spherical, isothermal distributions, the first and most important part of a galaxy lens model is an isothermal term. Variations on this can add ellipticity and/or shear into the mass distribution, or into the lens potential itself (Kochanek, 1991; Kassiola \& Kovner, 1993; Kormann et al., 1994; Keeton et al., 1997; Witt and Mao, 1997; Keeton and Kochanek, 1998). The latter set of models are generally much easier to deal with analytically, so we will concentrate on those.

Witt \& Mao (1995) showed that for a binary microlensing system, when the image multiplicity is a maximum (five in this case), then the sum of the signed magnifications of all 5 images is always unity. The sign of the magnification of an image is merely the parity of the image, +1 for ordinary images and -1 for inverted images. (see Schneider, Ehlers \& Falco (SEF), 1992 for a thorough review of lens theory and terminology.) Writing $\mu_{i}$ as the signed magnification of the $i^{t h}$ image, let us define $I \equiv \sum_{i} \mu_{i}$. This is a different quantity from the total magnification, which is given by $\sum_{i}\left|\mu_{i}\right|$. Witt \& Mao showed that $I=1$, independent of quantities such as the Einstein radii of the lenses or the position of the source, as long as the source was inside any of the caustics. Witt \& Mao ingeniously applied resultant theory to the lens equations to obtain this result. More recently, Rhie (1997) used a coordinate transformation to derive the same result, $I=1$, much more easily, and to show that $I=1$ for $\mathrm{N}$ point masses in a plane acting as a gravitational lens, for all nonnegative $\mathrm{N}$. Thus, for microlensing, the quantity $I$ is an invariant, independent of lens model, so we will refer to it as the "magnification invariant". The invariance of the sum of the signed magnifications of the maximum number of images is useful for microlensing because, even though individual images cannot be resolved, the total magnification is measurable, and conditions upon the sum of the signed magnification imply conditions upon the sum of the unsigned magnifications, as Witt \& Mao describe. Galaxy lensing presents us with the reverse situation - although the total magnification cannot be determined observationally, individual images can be resolved. Since for many lenses, it is easy to assign image parities, one can compute the sum of the signed fluxes. Therefore, if for galaxy lensing there are constraints upon the sum of the signed fluxes, as there are for microlensing, these constraints should be observationally verifiable. It is therefore interesting to ask whether such constraints exist for models of galaxy lenses. In this letter, we consider several of the commonly used models for galaxy lenses, and show that for many of them, although not all, such a property is true. We then apply this knowledge to several observed lens systems, and show how the magnification invariant can be used to determine properties of the lens, without fitting.

\section{METHOD}

The method we employ is straightforward, and is based upon that used by Witt \& Mao (1995) for the binary point lens. For the simple lens potentials we consider, we write the lens equations as polynomials in the variables $\vec{x}$ and $\mu$, where $\vec{x}$ is the angular position and $\mu$ is the signed magnification. We then apply elimination theory to eliminate the image positions and obtain a single equation satisfied by all of the magnifications. Elimination theory is a well developed field within algebraic geometry, and there are multiple techniques and algorithms known to eliminate variables from simultaneous polynomial equations. The most popular method is to compute the Gröbner basis of the original set of polynomials. When lexicographic ordering of the variables is chosen, successive basis elements will have variables eliminated (Cox et al., 1997). A more classical technique is to compute the generalized resultant. Sylvester, in the 19th century, developed a formula to take the resultant of three polynomials in three variables (Gelfand et al., 1994), which is the category into which the lens equations fall. Whatever technique is used, the result is a single polynomial equation in the 
magnification, whose roots are the signed magnifications of the images. Thus, the degree of the final polynomial equals the maximum number of images. We should remark that it is not always possible to perform such a procedure for all lens models, and it is important to verify that the degree of the final polynomial does equal the maximum number of images. Since the sum of the roots of a polynomial equals the ratio of the second coefficient to the leading coefficient (up to a sign), to compute the sum of the magnifications, we need only compute this polynomial. This ratio of coefficients then gives us the magnification invariant. It is not even necessary to solve the lens equations! This technique was first set out by Witt and Mao in their 1995 paper on binary point lenses.

\section{LENS MODELS}

We now apply this technique to several popular galaxy lens models. Galaxy lens models, as stated earlier, generally consist of variations on the singular isothermal sphere (SIS). The lens potential for an ordinary SIS is $\psi=b r$, where $b$ is the Einstein radius (which sets the physical scale of the problem) and $r=\sqrt{x^{2}+y^{2}}$ is the distance to the center of the lens (SEF, 1992). Galaxies are not perfectly spherical, however, and thus ellipticity and/or shear terms are added to the lens potential to account for this (see Blandford \& Kochanek, 1987; Kochanek, 1991; Kassiola \& Kovner, 1993; Witt \& Mao, 1997). For the models we consider, we let $\gamma$ measure the strength of the shear, and $\epsilon$ be the ellipticity. We choose without loss of generality coordinates centered on the lens, and oriented along the ellipticity axis or direction of shear. The models are :

1. SIS + elliptical $: \psi=b r+\gamma b r \cos (2 \theta)$

2. SIE $: \psi=b R=b \sqrt{(1-\epsilon) x^{2}+(1+\epsilon) y^{2}}$

3. SIS + external shear: $\psi=b r+\frac{\gamma}{2} r^{2} \cos (2 \theta)$

4. SIE + external shear: $\psi=b R+\frac{\gamma}{2} r^{2} \cos 2\left(\theta-\theta_{\gamma}\right)$

Here, SIE stands for "singular isothermal ellipse". This is not the isothermal elliptical mass distribution described by Kassiola \& Kovner (1993) and Kormann et al. (1994), but is instead an elliptical potential, corresponding to the singular isothermal elliptical potential (SIEP) of Kassiola \& Kovner (1993). In model 4 , the parameter $\theta_{\gamma}$ describes the orientation of the external shear relative to the ellipticity axes. We consider here only singular models since lensed systems generally consist of even numbers of images
(Kochanek, 1991), but later we will discuss one nonsingular model.

As an example of the procedure, we calculate $I$ for model 1, SIS+elliptical. For convenience, we use an orthonormal basis $\left\{e_{\hat{r}}, e_{\hat{\theta}}\right\}=\left\{\frac{\partial}{\partial r}, \frac{1}{r} \frac{\partial}{\partial \theta}\right\}$. In polar coordinates, with the source at $\left(s, \theta_{s}\right)$ and image at $(r, \theta)$, the time delay becomes

$$
\tau=\frac{1}{2}\left(r^{2}+s^{2}-2 r s \cos \left(\theta-\theta_{s}\right)\right)-b r-\gamma b r \cos (2 \theta)
$$

Stationarity of the time delay gives us the lens equations, the solutions of which are the image positions:

$$
\begin{gathered}
\tau_{, \hat{r}}=\frac{\partial \tau}{\partial r}=r-s \cos \left(\theta-\theta_{s}\right)-b(1+\gamma \cos (2 \theta))=0 \\
\tau_{, \hat{\theta}}=\frac{1}{r} \frac{\partial \tau}{\partial \theta}=s \sin \left(\theta-\theta_{s}\right)+2 \gamma b \sin (2 \theta)=0
\end{gathered}
$$

Note that the $\hat{\theta}$ equation is independent of $r$ (Kassiola and Kovner, 1995). The components of the Hessian are

$$
\tau_{; \hat{i} \hat{j}}=\left(\begin{array}{cc}
1 & 0 \\
0 & 1-\frac{b}{r}+3 \gamma \frac{b}{r} \cos (2 \theta)
\end{array}\right) .
$$

where we use semicolons to denote covariant derivatives. Thus, the Jacobian determinant for this lens (see appendix) is

$$
\|J\|=1-\frac{b}{r}+3 \gamma \frac{b}{r} \cos (2 \theta),
$$

and of course the signed magnification is $\mu=1 /\|J\|$, which is to be evaluated at each image position (i.e. at all of the solutions of the lens equations).

We now write this equation, and the two lens equations, as polynomial equations. Let $u=e^{i \theta}$ and $z=e^{i \theta_{s}}$. Then the lens equations become

$$
\begin{gathered}
\gamma b u^{4}+\frac{s}{z} u^{3}+2(b-r) u^{2}+s z u+\gamma b=0 \\
\gamma b u^{4}+\frac{s}{2 z} u^{3}-\frac{s z}{2} u-\gamma b=0 \\
3 \gamma b \mu u^{4}+2(\mu r-r-b \mu) u^{2}+3 \gamma b \mu=0 .
\end{gathered}
$$

Eliminating $r$ and $u$, and assuming $\gamma \neq 0$, we obtain the desired fourth degree polynomial in $\mu$, which is too long to print here. Again, this polynomial must be fourth degree since there are at most four images. We note that the leading coefficient, $a_{4}$, and the cubic coefficient, $a_{3}$, are related by

$$
\begin{aligned}
a_{4}=-a_{3}= & \frac{16 b^{4} \gamma^{4}}{z^{4}}\left(3 b^{2} s^{4}\left(9-2 z^{4}+9 z^{8}\right) \gamma^{2}\right. \\
& \left.-s^{6} z^{4}-768 b^{4} s^{2} z^{4} \gamma^{4}+4096 b^{6} z^{4} \gamma^{6}\right),
\end{aligned}
$$


and so the sum of the roots of this polynomial equals $-a_{3} / a_{4}=1$. This fourth degree polynomial has either 2 or 4 real roots. When the source is inside the caustic, all roots are real and thus $I=\sum_{i} \mu_{i}=1$. When the source is outside the caustic, 2 roots merge and become a complex conjugate pair. The real roots correspond to visible images, while the complex roots correspond to spurious solutions to the lens equations, which are not visible images (Petters, 1993). Therefore, when the number of real images is the maximum possible (4 in this case), $I=1$.

Applying this procedure to all of the galaxy lens models, we obtain Table 1 . We should note that model 4 has the surprising property that when the shear is oriented along the ellipticity axis (i.e. $\theta_{\gamma}=0$ ) and $\gamma=\epsilon$, then the caustic shrinks to a single point, located behind the lens. Although there are only two images for this special case, the two magnifications sum to give the quantity listed in Table 1 .

\section{DISCUSSION}

We have shown that for several models commonly used to describe galaxy lenses, the predicted image magnifications must obey the condition that their sum equal a simple constant, independent of source position (while the source lies within the caustic) and often independent of model parameters. This has some interesting consequences. To begin, we note that it is easy to compute the value of this magnification invariant for a quad lens system, since the parities of the images alternate as one goes around the ring of images. Now consider, for example, the object CLASS 1608+656 (Keeton \& Kochanek, 1996; Myers et al., 1995), which is a promising candidate for measurement of the Hubble constant $H_{0}$. By inspection, we see that images $\mathrm{A}$ and $\mathrm{B}$ should have positive parity and that $\mathrm{C}$ and $\mathrm{D}$ should have neg-

Table 1: Magnification invariants for the models shown. Here, $r=\sqrt{x^{2}+y^{2}}, \theta=\tan ^{-1}(y / x)$, $R=\sqrt{(1-\epsilon) x^{2}+(1+\epsilon) y^{2}}$.

\begin{tabular}{lcc}
\hline Model & $\psi$ & $\sum_{i} \mu_{i}$ \\
\hline 0 SIS & $b r$ & 2 \\
1 SIS + elliptical & $b r+\gamma b r \cos (2 \theta)$ & 1 \\
2 SIE & $b R$ & 2 \\
3 SIS + external & $b r+\frac{\gamma}{2} r^{2} \cos (2 \theta)$ & $\frac{2}{1-\gamma^{2}}$ \\
4 SIE + external & $b R+\frac{\gamma}{2} r^{2} \cos 2\left(\theta-\theta_{\gamma}\right)$ & $\frac{2}{1-\gamma^{2}}$ \\
\hline
\end{tabular}

ative parity (using the naming scheme used by Keeton \& Kochanek). Relative to B, A has brightness $2.06 \pm 0.06, \mathrm{C}$ has $0.85 \pm 0.03$, and $\mathrm{D}$ has $0.26 \pm 0.03$. Thus, the magnification invariant for this system is $1.95 \pm 0.07$, multiplied by image B's unknown magnification factor. Now, Seitz \& Schneider (1994), applying the Raychaudhuri equation (see MTW, 1973 or Wald, 1984), showed that minima of the time delay are never demagnified, that is $\mu \geq 1$. Since $\mathrm{B}$ is a minimum, its magnification factor must be $\geq 1$. Therefore, we immediately see that model 1 cannot possibly reproduce CLASS $1608+656$, since to do so would require that image $B$ have magnification $0.51 \pm 0.02$. The other models considered here are not expressly forbidden, but are quite unlikely to succeed, since they would require image B to have a magnification factor barely above the minimum possible value. Thus we see, without the effort of modeling, that none of the simple models considered here have hope of recovering the positions and brightnesses of object CLASS 1608+656. One can either ignore the fluxes when fitting, if their accuracy is suspect (which should not be the case for CLASS 1608+656, as fluxes were measured in the radio), or one must resort to more complicated models. The problem, as Kochanek (1991) has emphasized, is that the complexity of model is limited by the small number of observables, which is only 9 for a quad lens. Note that the simple models here already have 5 or 7 parameters, so there are very few parameters that can be added before the problem becomes underconstrained. Of course, these remarks apply only for fitting on the image plane. When fitting on the source plane, the images are solutions to different lens equations, and thus the sum of their magnifications need not be anything in particular. Indeed, when images are near the critical curve, the sum of the magnifications can take on arbitrary values if the images do not have the same source, and so the magnification invariant would have little meaning. However, for purposes other than verifying that an object is in fact gravitationally lensed, one ususally fits in the image plane.

Now, CLASS $1608+656$ is a particularly difficult object to model, but what about other lensed systems? Consider the "Cloverleaf", H1413+117 (Keeton \& Kochanek, 1996; Yun et al., 1997). Using the $I$ band fluxes listed by Keeton \& Kochanek, which should be the most trustworthy, we find that the magnification invariant equals 0.07 times A's magnification factor. We shouldn't trust this very far however, 
since the invariant computed from the $R$ band fluxes would be negative! In any case, fitting any of the simple models to H1413+117 would require very large image magnification factors for a good fit, about 15 for model 1 or about 30 or higher for the others. This is using the $I$ band data; using the $R$ or $B$ data would give an even smaller invariant, and therefore require even greater image magnifications. Since the fluxes of the four images are all comparable, this means that all of the images would be required to lie very close to the critical curve, implying a tiny caustic with the source inside. Since the size of the caustic is determined by the magnitude of the shear/ellipticity, constraining the caustic to be small limits the range of these parameters significantly. Indeed, even though H1413+117 would seem to be an ideal object for fitting - one might go so far as to call it a "model" lens system - we were unable to fit any of the listed models satisfactorily to it, using the $\chi_{\mathrm{S}}^{2}$ error function described in Kochanek (1991), except for model 4. By satisfactory fit, we mean $\chi^{2} / N_{d o f} \simeq 1$. Model 4 was able to give a good fit due to the feature mentioned above, that when the external shear and ellipticity are related in a specific way, the caustic shrinks to a point, even though the ellipticity may be significant. This feature enables model 4 to attain the large magnifications necessary, while possessing the ellipticity required to fit the image geometry. The other models, which cannot be elliptical without having a large caustic, are thus doomed to failure. Our difficulty in fitting this object is not much of a revelation; previous efforts at fitting H1413+117 using simple models such as the ones discussed here are unable to recover the image brightnesses satisfactorily. For example, neither Kayser et al. (1990) nor Kochanek (1991) attempted to fit the brightnesses. Yun et al. (1997) do fit the flux ratios, using a variant of model 4 with extra parameters. Their model is similar enough to model 4 to possess the same "shrinking caustic" feature, and not surprisingly their best fit has a tiny (tangential) caustic barely enclosing the source QSO, with the associated large image magnifications of order 50. The prerequisite that the caustic be tiny for a good fit makes sense in terms of the magnification invariant.

H1413+117 also illustrates another use of the magnification invariant. By merely adding up four numbers, we immediately learn that the image magnifications must be large. Yun et al. point out that this means that the curvature of the time delay surface at the ring of images is small, meaning that the relative time delays between images must be small. We have seen, however, that the magnifications must be large for all models, and therefore the predicted relative time delays between images will be small, independent of model. This is useful knowledge for $H_{0}$ determination.

We have so far discussed only singular models. Lens potentials can be rendered nonsingular by the introduction of a core radius. Altering the models discussed here to make them nonsingular destroys the invariance of the sum of the magnifications. However, the size of the core radius term is limited by the absence of a central image from observed lens systems. For small enough core radius the sum of the magnifications of the remaining four images will be close to the value for the singular model. Thus, our results should still apply for the cases of interest, where no central image is observed. For models based upon elliptical mass distributions, one can see from numerical calculations that the sum of the magnifications of the maximum number of images is not a constant. However, for typical parameter values, such as in Jackson et al. (1998), Myers et al. (1995) or Nair (1998), we find that the sum of the magnifications of four images is between 2 and 3 , so this model should be marginally better at fitting objects like CLASS 1608+656. Models with multiple galaxies (e.g. Hogg \& Blandford, 1994) can have a much higher magnification invariant, however they have a maximum number of images not equal to four.

The magnification invariant, we have seen, is easy to calculate for observed quad lenses, since one merely adds up four numbers. Doing so gives us useful information on the ability of lens models to fit the observations, even before fitting. We have seen that for small invariants, such as H1413+117, large magnifications are necessary. On the other hand, invariants that are large, such as for CLASS $1608+656$, can help rule out models a priori. It is interesting that two lenses recently discovered, B1933+503 (Sykes et al., 1997) and B0712+472 (Jackson et al., 1998) both have large invariants and should, like CLASS $1608+656$, be hard to fit using the simple models. We hope that models based on realistic mass distributions in galaxies, e.g. Keeton \& Kochanek (1998), willl fare better at describing these objects. 


\section{ACKNOWLEDGEMENTS}

The author would like to thank John Wavrik for discussions of elimination theory, Jeffrey Rabin and Roger Blandford for many helpful conversations, and Kim Griest for encouragement, discussions, and for reading the manuscript. This work was supported in part by the US DOE under grant DEFG0390ER 40546.

\section{APPENDIX}

In an arbitrary frame, it is not obvious how the Jacobian of the lens mapping is related to the Hessian of the time delay. We can easily relate these two by noting that in the usual Cartesian coordinates, $J^{i}{ }_{j}=\tau^{; i}{ }_{j}$, i.e. that the components of the Jacobian matrix equal the components of the Hessian. Note, however, that the Hessian in this equation has been contracted with the metric tensor, since the Hessian is a $\left(\begin{array}{l}0 \\ 2\end{array}\right)$-tensor while the Jacobian, a linear transformation taking 1-forms to 1-forms, must be a $\left(\begin{array}{l}1 \\ 1\end{array}\right)$-tensor. Of course, in orthonormal frames such as the one used above, or Cartesian coordinates, the metric tensor is merely the identity matrix. Knowing the relation between the Jacobian and Hessian in Cartesian coordinates tells us their relationship in arbitrary frames. Let $d x^{\hat{i}}$ be the 1 -form dual to the basis vector $e_{\hat{i}}$ in the image plane, and similarly for $d s^{\hat{i}}$ in the source plane. Then

$$
d s^{\hat{i}}=\frac{\partial s^{\hat{i}}}{\partial s^{j}} \frac{\partial s^{j}}{\partial x^{k}} \frac{\partial x^{k}}{\partial x^{\hat{l}}} d x^{\hat{l}}
$$

where unhatted components are Cartesian, and hatted components are in arbitrary frames. Substituting the Cartesian lensing Jacobian

$$
\frac{\partial s^{j}}{\partial x^{k}}=\tau^{; j}{ }_{k}=\frac{\partial x^{j}}{\partial x^{\hat{m}}} \tau^{; \hat{m}}{ }_{\hat{n}} \frac{\partial x^{\hat{n}}}{\partial x^{k}}
$$

into the previous equation gives us the lensing Jacobian expressed in terms of the Hessian computed in an arbitrary frame.

$$
J_{\hat{l}}^{\hat{i}}=\frac{\partial s^{\hat{i}}}{\partial s^{j}} \frac{\partial x^{j}}{\partial x^{\hat{k}}} \tau^{i \hat{k}}{ }_{\hat{\imath}}
$$

For orthonormal frames, the Jacobian matrices $\frac{\partial s^{\hat{i}}}{\partial s^{j}}$ and $\frac{\partial x^{j}}{\partial x^{k}}$ are merely rotation matrices, with unit determinant, so that the Jacobian determinant equals the determinant of the Hessian matrix.

\section{REFERENCES}

Alcock, C. et al. (MACHO collaboration), 1997, ApJ, 479,119

Alcock, C. et al. (MACHO collaboration), 1998, astro-ph/9807163

Blandford, R.D. \& Kochanek, C.S., 1987, ApJ, 321, 658

Blandford, R.D. \& Narayan, R. 1986, ApJ, 310, 568

Cox, D., Little, J. \& O'Shea, D., 1992, Ideals, Varieties, and Algorithms. Springer-Verlag, New York

Gelfand, I.M., Kapranov, M.M. \& Zelevinsky, A.V., 1994, Discriminants, Resultants and Multidimensional Determinants. Birkhäuser, Boston. p. 118.

Hogg, D. W. \& Blandford, R. D., 1994, MNRAS, 268, 893

Jackson, N. et al., 1998, MNRAS, 296, 483

Kassiola, A. \& Kovner, I., 1993, ApJ, 417, 450

Kassiola, A. \& Kovner, I., 1995, MNRAS, 272, 363

Kayser, R. et al., 1990, ApJ, 364, 15

Keeton, C.R \& Kochanek, C. S., 1996, in Kochanek, C.S. \& Hewitt, J.N. (eds.), IAU 173, Melbourne, Astrophysical Applications of Gravitational Lensing, Kluwer, p. 149

Keeton, C.R, Kochanek, C. S. \& Seljak, U., 1997, ApJ, 482, 604

Keeton, C.R \& Kochanek, C. S., 1998, ApJ, 495, 157

Kochanek, C. S., 1991, ApJ, 373, 354

Kormann, R., Schneider, P. \& Bartelmann, M, 1994, Astron. Astrophys., 284, 285

Misner, C.W., Thorne, K.S. \& Wheeler, J.A., 1973, Gravitation. W.H. Freeman \& Co., New York. p. 582 (MTW)

Myers, S. et al., 1995, ApJ, 447, L5

Nair, S., 1998, astro-ph/9803076

Petters, A., 1993, J. Math. Phys., 34, 3555

Rhie, S. H., 1997, ApJ, 484, 63 
Schneider P., Ehlers J., Falco E. E., 1992, Gravitational Lenses. Springer-Verlag, New York. (SEF)

Seitz, S. \& Schneider, P., 1994, Astron. Astrophys., 287,349

Sykes, C.M. et al., 1997, astro-ph/9710358

Wald, R.M., 1984, General Relativity. University of Chicago Press, Chicago. p. 218

Witt, H. J \& Mao, S., 1995, ApJ, 447, L105

Witt, H. J \& Mao, S., 1997, MNRAS, 291, 211

Yun, M.S. et al., 1997, ApJ, 479, L9

This 2-column preprint was prepared with the AAS IATEX macros v4.0. 